\title{
Paraneoplastic limbic encephalitis in a patient with extensive disease small-cell lung cancer
}

\author{
SEBASTIAN OCHENDUSZKO ${ }^{1}$, BARTOSZ WILK $^{2}$, JOANNA DABROWSKA $^{1}$, \\ IZABELA HERMAN-SUCHARSKA ${ }^{3}$, ANNA DUBIS ${ }^{3}$ and MIROSLAWA PUSKULLUOGLU ${ }^{4}$ \\ ${ }^{1}$ Department of Oncology, University Hospital in Krakow, 31-531 Krakow; ${ }^{2}$ Department of Oncology, \\ Medical University of Silesia Hospital No. 7, Professor Leszek Giec Upper Silesian Medical Centre, \\ 40-635 Katowice; ${ }^{3}$ Department of Electroradiology, Jagiellonian University Medical College, 31-126 Krakow; \\ ${ }^{4}$ Department of Oncology, Jagiellonian University Medical College, 31-531 Krakow, Poland
}

Received August 10, 2016; Accepted November 22, 2016

DOI: $10.3892 / \mathrm{mco} .2017 .1162$

\begin{abstract}
Paraneoplastic limbic encephalitis (PLE) is a rare disorder infrequently accompanying malignancy, coexisting in $~ 50 \%$ of the cases with small-cell lung cancer (SCLC). The pathomechanism of PLE is considered to be immune-mediated, with production of specific anti-Hu antibodies and activation of T-cells directed against onconeural antigens present on both tumor cells and neurons. We herein report the case of a 50-year-old male patient who, prior to being diagnosed with SCLC, presented with typical symptoms of PLE (seizures, subacute cognitive dysfunction with severe memory impairment, anxiety and hallucinations). The initial brain magnetic resonance imaging examination revealed mild enlargement and hyperintensity of the hippocampal gyri bilaterally, with narrowed temporal horns of the lateral ventricles; the findings of the cerebrospinal fluid examination were compatible with the diagnosis of lymphocytic meningitis. Due to the suspected infectious origin of the disease, treatment with acyclovir and antibiotics was initially applied. However, following subsequent diagnosis of the underlying SCLC and the presence of antineuronal anti-Hu antibodies in the patient's serum, the diagnosis of PLE accompanying extensive-disease (ED) SCLC was confirmed. In addition to the standard cytotoxic therapy, throughout the course of his disease the patient also continued treatment with valproic acid (VPA) as prophylaxis for the initial seizures. VPA is known to be a potent histone deacetylase inhibitor that may reverse epigenetic changes in tumor cells and potentially improve the outcome of cancer patients. The patient succumbed to the disease 25 months after the diagnosis of malignancy; such a long course is observed in
\end{abstract}

Correspondence to: Dr Sebastian Ochenduszko, Department of Oncology, University Hospital in Krakow, 10 Sniadeckich Street, 31-531 Krakow, Poland

E-mail: sebaochenduszko@gmail.com

Key words: small-cell lung cancer, paraneoplastic limbic encephalitis, anti-Hu antibodies, chemotherapy, valproic acid, synergistic effect only 5\% of patients with ED SCLC. Therefore, it was hypothesized that the accompanying paraneoplasia and treatment with VPA may have improved the outcome in this patient.

\section{Introduction}

An estimated 1.8 million new lung cancer cases occurred in 2012, accounting for $\sim 13 \%$ of total cancer diagnoses, making lung cancer the most frequently diagnosed malignancy and leading cause of cancer-related mortality among men worldwide and among women in more developed countries (1). Small-cell lung cancer (SCLC) accounts for only 13\% of all lung cancers and has a very poor prognosis, with $\sim 5 \%$ of patients with extensive-disease (ED) SCLC surviving for 2 years (2). Although paraneoplastic syndromes are fairly common in SCLC (3), it is estimated that $<5 \%$ of patients with SCLC will develop a clinically significant paraneoplastic neurological disorder (PND) (4). Paraneoplastic limbic encephalitis (PLE) is a rare disorder infrequently accompanying cancer, coexisting with SCLC in $~ 50 \%$ of the cases (5). It was suggested that the prognosis of cancer patients with accompanying paraneoplastic syndromes may be better compared with that of patients with the same cancer but without associated paraneoplasia (6-8).

Over the last few years, a major advancement in understanding SCLC biology has been made (9), which may finally lead to the introduction of new drugs and improvement of the treatment efficacy. Preclinical data demonstrated that the addition of valproic acid (VPA), a potent histone deacetylase inhibitor, to standard chemotherapy regimens in SCLC, may improve patient outcome $(10,11)$.

We herein report on the case of a patient with ED SCLC who developed PLE during the course of his disease, with symptoms of PLE typically preceding the diagnosis of SCLC. The diagnostic process of the SCLC and the PLE in this patient were also described and the potential synergistic effect of the VPA and chemotherapy in the treatment of the SCLC was investigated.

\section{Case report}

A 50-year-old man with no medical history was admitted to the community hospital in July, 2007 with generalized seizures. 
The patient was a heavy cigarette smoker ( 70 pack-years) and had a history of alcohol abuse. The family history was insignificant. On admission, the computed tomography (CT) scan of the brain revealed no abnormalities, but the chest X-ray showed enlarged right hilar nodes. The blood count, liver and renal function tests were within normal limits. Treatment with VPA and dexamethasone was initiated. Two days later, the patient developed symptoms of acute psychosis and was referred to the Department of Psychiatry at the University Hospital in Krakow. Finally, due to fever (temperature up to $39.6^{\circ} \mathrm{C}$ ) and suspicion of central nervous system infection, the patient was transferred to the Department of Infectious Diseases. On physical examination, the patient was febrile and conscious; however, he was somnolent with significant short-term memory loss and capable of responding only to basic questions. No focal neurological deficits or neck rigidity were present. The results of the cerebrospinal fluid (CSF) examination (colour, yellowish; clarity, muddy; cytosis, 13 cells $/ \mu 1$; protein content, $0.49 \mathrm{~g} / 1$; glucose, $2.3 \mathrm{mmol} / 1$; chloride, $127 \mathrm{mmol} / \mathrm{l}$ ) were compatible with the diagnosis of lymphocytic meningitis. The magnetic resonance imaging (MRI) of the brain revealed a mild enlargement and hyperintensity of the hippocampal gyri bilaterally, with narrowed temporal horns of the lateral ventricles (Fig. 1). The CSF cultures and the serological test for Lyme disease were both negative. The chest and abdominal CT scan revealed a $10 \times 5-\mathrm{cm}$ neoplastic mass in the mediastinum, enlarged right hilar, subcarinal, paratracheal and left hilar lymph nodes, as well as a right hydrothorax. The biopsy of the enlarged right hilar nodes was positive for SCLC.

After a course of acyclovir and antibiotics (ceftriaxone, rifampicin, isoniazid, imipenem/cilastatin and linezolid), the patient was subsequently referred to the Department of Oncology for further treatment of the ED SCLC. First-line palliative chemotherapy with cisplatin and etoposide was decided. At that moment, based on the combination of the clinical picture (seizures, personality changes, short-term memory loss, pathological voracity), the MRI results and the presence of SCLC, PLE accompanying the SCLC was suspected. PLE was subsequently confirmed by the presence of anti-Hu autoantibodies in the patient's serum. Treatment with corticosteroids was continued, with the aim of suppressing the immunological system response and reducing the symptoms of the paraneoplastic syndrome. After 6 cycles of chemotherapy, having achieved a partial response to the treatment, the patient underwent prophylactic cranial irradiation (30 Gy in 15 fractions). The subsequent brain MRI showed less prominent hyperintensity on $\mathrm{T} 2$-weighted images in the hippocampal gyri bilaterally, with signs of limbic system atrophy (Fig. 2). There was no amelioration of the brain functions after completion of these therapies, except for a mild improvement of the patient's memory.

Five months later, a control CT scan revealed tumor progression and the patient received 6 cycles of second-line chemotherapy with cyclophosphamide, doxorubicin and vincristine (CAV regimen). At the next progression, the patient was treated with palliative radiotherapy for the tumor in the chest and cyclophosphamide monotherapy. Finally, the patient succumbed to the disease 25 months after the diagnosis of the ED SCLC.

\section{Discussion}

The clinical diagnosis of PLE may be challenging, as its typical symptoms (subacute cognitive dysfunction with severe memory impairment, seizures and psychiatric features including depression, anxiety and hallucinations) may be similar to those observed in other cancer-related complications, metabolic and toxic encephalopathies, infections (particularly herpes simplex encephalitis) and to the side effects of cancer therapy $(5,12-14)$. In addition, the presenting symptoms may differ from those considered typical of the syndrome and the majority of the patients are not known to have cancer (5). However, fulfilling all the following criteria for PLE may facilitate its diagnosis: i) A compatible clinical picture; ii) an interval of $<4$ years between the onset of neurological symptoms and cancer diagnosis; iii) exclusion of other cancer-related complications (metastasis, infection, metabolic and nutritional deficits, cerebrovascular disorder or side effects of therapy) that may cause symptoms of limbic dysfunction; iv) at least one of the following: CSF with inflammatory changes, MRI showing unilateral or bilateral temporal lobe abnormalities on T2-weighted images or atrophy on T1-weighted images, and an electroencephalogram showing slow- or sharp-wave activity in one or both temporal lobes (5).

MRI examination of the brain and the spinal cord are required to exclude other causes of the neurological symptoms, including cancer metastases. The typical initial MRI findings in PLE [common FLAIR hyperintensity in medial temporal lobes (15)] may be similar to the ones observed in other forms of encephalitis of the limbic system, particularly during the early stages of herpes simplex encephalitis. However, patients with herpes simplex encephalitis usually display distinct characteristics: Prominent edema and mass effect involving one or both inferior-medial temporal lobes, the inferior frontal lobes and the cingulate gyrus, gyral enhancement in the majority of cases and, frequently, signs of hemorrhage $(5,16)$.

The CSF examination in a patient with PLE is often abnormal, with mild to moderate pleocytosis, increased protein content, intrathecal synthesis of IgG and oligoclonal bands, suggesting the presence of an inflammatory or immune-mediated disorder $(4,5)$.

The majority of the paraneoplastic syndromes involving the nervous system are currently considered to be immune-mediated (17). Ectopic expression by a tumor of the antigens normally present in the neurons (referred to as onconeural antigens) induces an immune system response with production of specific antibodies and activation of T-cells that, after crossing the blood-brain barrier, react with the onconeural antigens and produce specific neurological symptoms (17). Therefore, the majority of patients with paraneoplastic syndromes involving the nervous system have detectable antineuronal antibodies in their serum and/or the CSF $(4,5)$ and their presence may facilitate the diagnosis of the paraneoplastic syndrome and allow early detection of the associated tumor (12). Anti-Hu antibodies are typically found in SCLC, neuroblastoma and prostate cancer (17). SCLC patients with anti-Hu antibodies have a lower probability of improvement in PLE symptoms compared with patients without anti-Hu antibodies (12), and only a minority of these patients responds to treatment with corticosteroids, intravenous immunoglobulins or plasma exchange (18). 

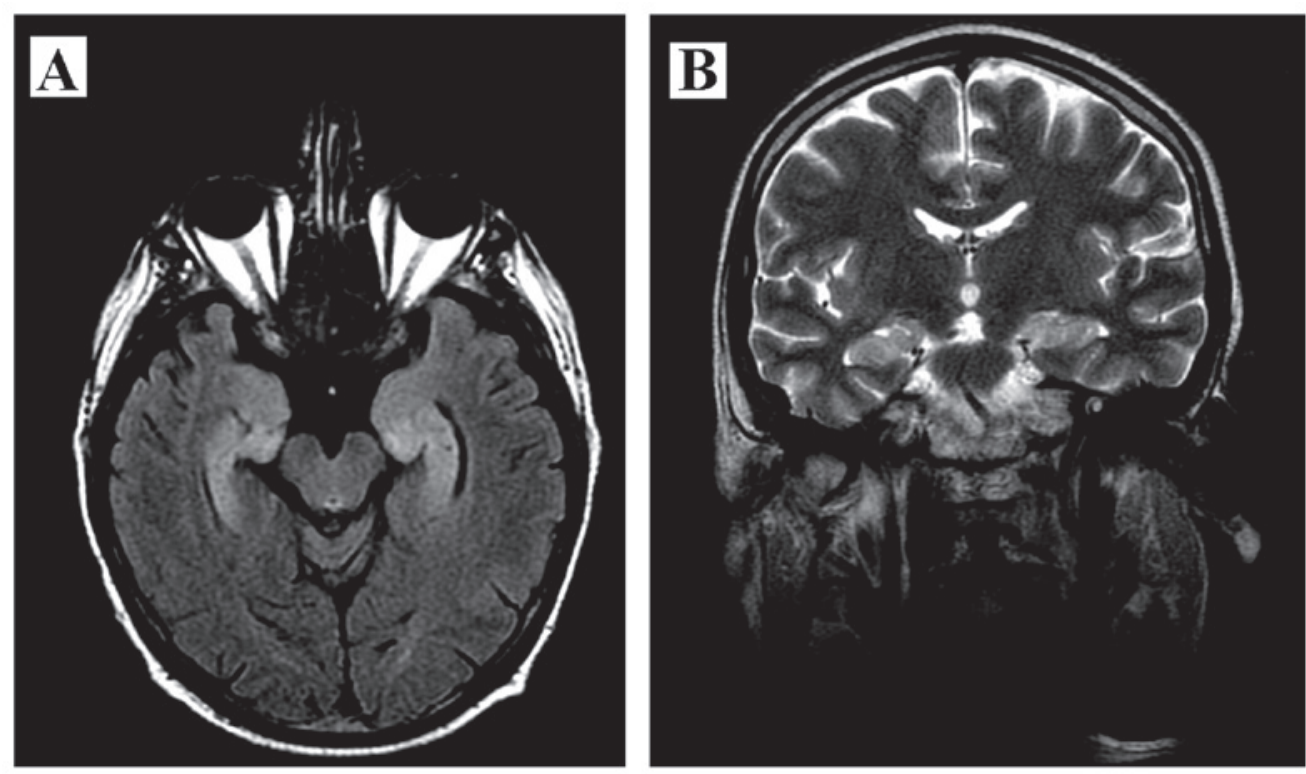

Figure 1. Pre-treatment brain magnetic resonance imaging examination. (A) FLAIR T2 sequence, axial plane; (B) frFSET2 sequence, coronal plane. Mild enlargement and hyperintensity of the hippocampal gyri bilaterally and narrowed temporal horns of the lateral ventricles were observed.
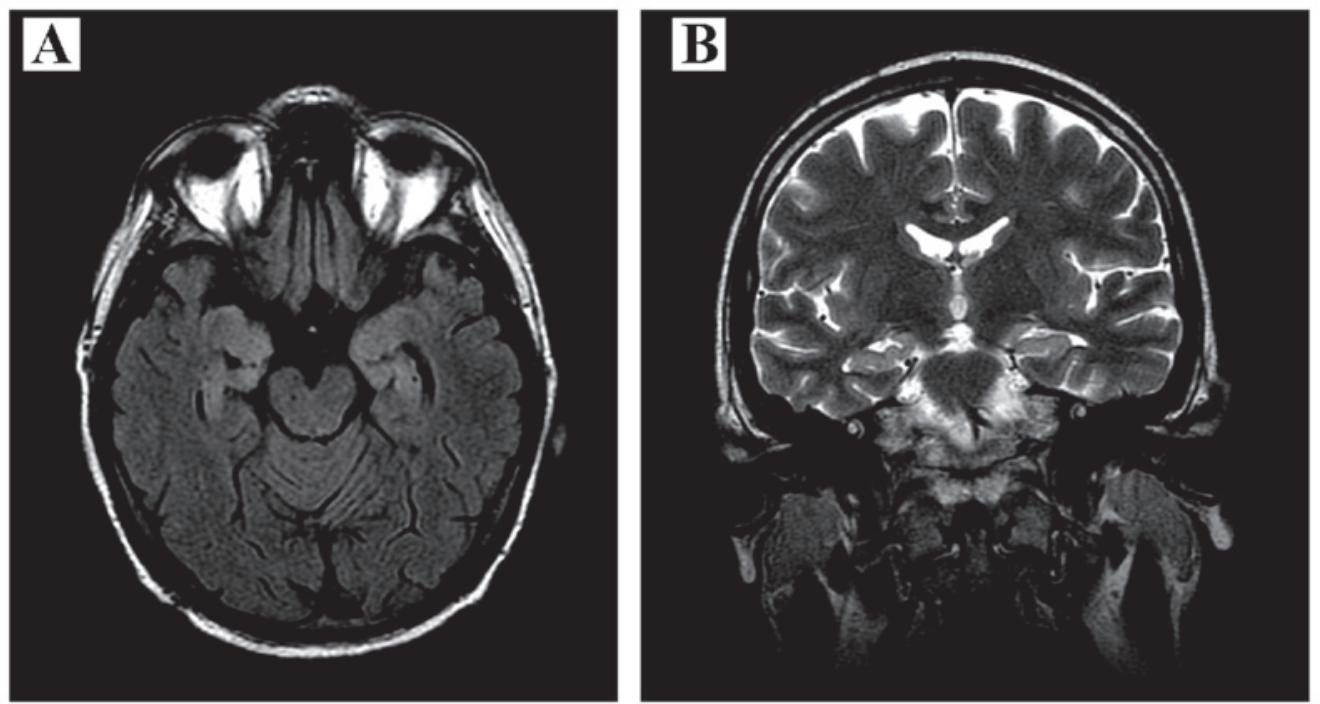

Figure 2. Post-treatment brain magnetic resonance imaging (MRI) examination. (A) FLAIR T2 sequence, axial plane; (B) frFSET2 sequence, coronal plane. Less prominent hyperintensity on T2-weighted images in the hippocampal gyri bilaterally, with signs of limbic system atrophy. Compared with the first MRI, discrete widening of both temporal horns of the lateral ventricles was observed.

With the currently available therapy, the median survival of patients with ED SCLC is 6-12 months, with an overall survival of $<5 \%$ after 2 years $(2,10)$. The prolonged survival in our patient may result from several factors. First, the prognosis of cancer patients with a paraneoplastic syndrome appears to be better compared with that of patients with the same malignancy but without the associated paraneoplastic disorder (6-8). It is considered that the immune response triggered by onconeural antigens not only injures a specific part of the nervous system and is responsible for the clinical presentation of the paraneoplastic syndrome, but may also control tumor growth (8). Second, the chronic use of VPA may have improved the outcome in this patient. VPA is a potent histone deacetylase inhibitor and may affect the dynamic structure of chromatin and the transcriptional processes leading to the modulation of pathways involved in cell cycle and apoptosis $(10,11,19)$. Moreover, preclinical data demonstrated that the combination of VPA and standard chemotherapy regimens in SCLC may exert a synergistic antitumor effect $(10,11)$.

In conclusion, the case presented herein underlines the need for precise evaluation of all cancer patients presenting with symptoms of a paraneoplastic disorder. These symptoms usually precede the diagnosis of the cancer itself and may facilitate the diagnosis of the underlying malignancy. Moreover, this case suggests that the efficacy of the standard chemotherapy regimens in SCLC may be further improved by additional agents, such as VPA, which may reverse epigenetic changes in cancer cells. 


\section{Acknowledgements}

The authors would like to thank Joanna Gołąb, the language editor, for her help in the preparation of this manuscript.

\section{References}

1. Torre LA, Bray F, Siegel RL, Ferlay J, Lortet-Tieulent J and Jemal A: Global Cancer Statistics, 2012. CA Cancer J Clin 65: 87-108, 2015.

2. Puglisi M, Dolly S, Faria A, Myerson JS, Popat S and O'Brien ME: Treatment options for small cell lung cancer-do we have more choice? Br J Cancer 102: 629-638, 2010.

3. Amini A, Byers LA, Welsh JW and Komaki RU: Progress in the management of limited-stage small cell lung cancer. Cancer 120: 790-798, 2014.

4. Bataller L and Dalmau J: Paraneoplastic neurologic syndromes. Neurol Clin 21: 221-247, ix, 2003.

5. Gultekin SH, Rosenfeld MR, Voltz R, Eichen J, Posner JB and Dalmau J: Paraneoplastic limbic encephalitis: Neurological symptoms, immunological findings and tumour association in 50 patients. Brain 123: 1481-1494, 2000.

6. Altman AJ and Baehner RL: Favorable prognosis for survival in children with coincident opso-myoclonus and neuroblastoma. Cancer 37: 846-852, 1976.

7. Maddison P, Newsom-Davis J, Mills KR and Souhami RL: Favourable prognosis in Lambert-Eaton myasthenic syndrome and small-cell lung carcinoma. Lancet 353: 117-118, 1999.

8. Graus F, Dalmou J, Reñé R, Tora M, Malats N, Verschuuren JJ, Cardenal F, Viñolas N, Garcia del Muro J, Vadell C, et al: Anti-Hu antibodies in patients with small-cell lung cancer: Association with complete response to therapy and improved survival. J Clin Oncol 15: 2866-2872, 1997.

9. Altan M and Chiang AC: Management of small cell lung cancer: Progress and Updates. Cancer J 21: 425-433, 2015.

10. Hubaux R, Vandermeers F, Crisanti MC, Kapoor V, Burny A, Mascaux C, Albelda SM and Willems L: Preclinical evidence for a beneficial impact of valproate on the response of small cell lung cancer to first-line chemotherapy. Eur J Cancer 46: 1724-1734, 2010
11. Hubaux R, Vandermeers F, Cosse JP, Crisanti C, Kapoor V, Albelda S, Mascaux C, Delvenne P, Hubert P and Willems L: Valproic acid improves second-line regimen of small cell lung carcinoma in preclinical models. ERJ Open Res 1: 00028-2015, 2015.

12. Alamowitch S, Graus F, Uchuya M, Reñé R, Bescansa E and Delattre JY: Limbic encephalitis and small cell lung cancer. Clinical and immunological features. Brain 120: 923-928, 1997.

13. Newman NJ, Bell IR and McKee AC: Paraneoplastic limbic encephalitis: Neuropsychiatric presentation. Biol Psychiatry 27: 529-542, 1990.

14. Bakheit AM, Kennedy PG and Behan PO: Paraneoplastic limbic encephalitis: Clinico-pathological correlations. J Neurol Neurosurg Psychiatry 53: 1084-1088, 1990.

15. Dalmau J and Rosenfeld MR: Paraneoplastic syndromes of the CNS. Lancet Neurol 7: 327-340, 2008.

16. Demaerel P, Wilms G, Robberecht W, Johannik K, Van Hecke P, Carton $\mathrm{H}$ and Baert AL: MRI of herpes simplex encephalitis. Neuroradiology 34: 490-493, 1992.

17. Darnell RB and Posner JB: Paraneoplastic syndromes involving the nervous system. N Engl J Med 349: 1543-1554, 2003.

18. Graus F, Keime-Guibert F, Reñe R, Benyahia B, Ribalta T, Ascaso C, Escaramis G and Delattre JY: Anti-Hu-associated paraneoplastic encephalomyelitis: Analysis of 200 patients. Brain 124: 1138-1148, 2001.

19. Duenas-Gonzalez A, Candelaria M, Perez-Plascencia C, Perez-Cardenas E, de la Cruz-Hernandez E and Herrera LA: Valproic acid as epigenetic cancer drug: Preclinical, clinical and transcriptional effects on solid tumors. Cancer Treat Rev 34: 206-222, 2008 